Pacific Journal of Mathematics

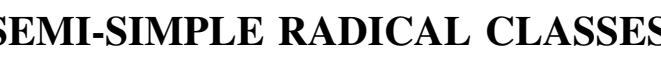




\section{SEMI-SIMPLE RADICAL CLASSES}

\section{PATRICK N. StewarT}

\section{The purpose of this paper is to characterize all semi-simple} radical classes (those classes of rings which are semi-simple classes and at the same time radical classes).

Andrunakievic has shown that the class of Boolean rings is a semisimple radical class. More recently, Armendariz has considered such classes.

For " $I$ is an ideal of the ring $R$ " we shall write " $I \triangleleft R$ ".

Following Divinsky [6], but substituting classes of rings for ring properties, we define:

(i) A nonempty class of rings $\mathscr{C}$ is a radical class if and only if $\mathscr{C}$ satisfies the following conditions:

(A) Homomorphic images of rings in $\mathscr{C}$ are in $\mathscr{C}$.

(B) Every ring $R$ has an ideal $\mathscr{C}(R) \in \mathscr{C}$ such that if $I \triangleleft R$ and $I \in \mathscr{C}$ then $I \subseteq \mathscr{C}(R)$.

(C) The only ideal of the factor ring $R / \mathscr{C}(R)$ which is in $\mathscr{C}$ is the zero ideal.

(ii) If $\mathscr{C}$ is a radical class, a ring $R$ is $\mathscr{C}$ semi-simple if and only if $\mathscr{C}(R)=(0)$.

(iii) A nonempty class of rings $\mathscr{C}$ is a semi-simple class if and only if $\mathscr{C}$ satisfies the following conditions:

(E) Every nonzero ideal of a ring in $\mathscr{C}$ can be homomorphically mapped onto a nonzero ring in $\mathscr{C}$.

(F) If every nonzero ideal of a ring $R$ can be homomorphically mapped onto a nonzero ring in $\mathscr{C}$ then $R \in \mathscr{C}$.

2. Rings without nilpotent elements. Our purpose in this section is to establish:

THEOREM 2.1.1 A ring $R$ without nilpotent elements is isomorphic (to a subdirect sum of rings without proper divisors of zero.

It will be convenient to first prove:

LEMma 2.2. If $R$ has no nilpotent elements and $0 \neq x \in R$ then

(i) $x_{r}=\{y \in R: x y=0\} \triangleleft R$ and $x_{r}=x_{l}=\{y \in R: y x=0\}$,

(ii) $x \notin x_{l}$,

1 The author wishes to thank the referee for pointing out that this result has also been obtained by V. Andrunakievic and Ju. M. Rjabuhin, Rings without nilpotent elements, and completely simple ideals, Dokl. Akad. Nauk. SSR. 180, 9-11 (Translation, Soviet Mathematics 9 (1968), 565-568). 
(iii) if $r \in R$ and $r x \in x_{l}$ then $r \in x_{l}$,

(iv) the factor ring $R / x_{l}$ has no nilpotent elements.

Proof. Let $R$ be a ring with no nilpotent elements and $0 \neq x \in R$. If $a \in R$ and $a x=0$ then $(x a)^{2}=0$ so $x a=0$. Similarily if $x a=0$ then $a x=0$. This establishes (i). Since $x^{2} \neq 0$, (ii) is clear. If $a$, $b \in R$ and $a b^{2}=0$ then $(b a b)^{2}=0$ so $b a b=0$, but then $(a b)^{2}=0$ so $a b=0$. From this (iii) and (iv) follow immediately.

To prove the theorem it is sufficient to find, for each $0 \neq x \in R$, an ideal $I(x)$ of $R$ for which $R / I(x)$ has no proper divisors of zero and $x \notin I(x)$. Let $Z(x)=\{I \triangleleft R: x \notin I$, if $r x \in I$ then $r \in I$, and $R / I$ has no nilpotent elements\}. By $2.2 x_{\iota} \in Z(x)$ so $Z(x) \neq \varnothing$ and it is clear that the union of an ascending chain in $Z(x)$ is also in $Z(x)$. Thus we may choose, by Zorn's Lemma, $I(x)$ maximal in $Z(x)$.

If $a \in R$ and $a \in I(x)$ let $J=\{y \in R: a y \in I(x)\} \supseteqq I(x)$. Then $J / I(x)=$ $(a+I(x))_{r}$ in $R / I(x)$ and by 2.2 (i) $(a+I(x))_{l}=(a+I(x))_{r} \triangleleft R / I(x)$. Since $a \notin I(x), a x \notin I(x)$ so $x \notin J$. If $r x \in J$ then $a r x \in I(x)$ so $a r \in I(x)$, hence $r \in J$. Finally by 2.2 (iv) $R / J \cong R / I(x) / J / I(x)$ has no nilpotent elements, so $J \in Z(x)$. Hence $J=I(x)$ so $R / I(x)$ has no proper divisors of zero.

Note 2.3. The generalized nil radical $\mathrm{Ng}$ of Andrunakievic [4] and Thierrin [10] (see also [6]) is the upper radical with respect to the class of rings without proper divisors of zero. A ring $R$ is $N g$ semi-simple if and only if $R$ is isomorphic to a subdirect sum of rings without proper divisors of zero. In this context, 2.1 can be restated as: A ring $R$ is $N g$ semi-simple if and only if $R$ has no nilpotent elements.

3. $\mathscr{B}_{1}$-rings. If $x \in R$, let $[x]=$ the subring of $R$ generated by $x$.

Definition 3.1. $R$ is a $\mathscr{B}_{1}$-ring.$\equiv$. for all $x \in R,[x]=[x]^{2}$.

Let $R$ be a ring and $x \in R$. Clearly $[x]=[x]^{2}$ if and only if $x \in[x]^{2}$ if and only if there are integers $a_{2}, \cdots, a_{k}$ such that $x=\sum_{i=2}^{k} a_{i} x^{i}$. Using this it is clear that homomorphic images of $\mathscr{B}_{1}$-rings are $\mathscr{B}_{1}$ rings and that if $A / B$ and $B$ are $\mathscr{B}_{1}$-rings then $A$ is a $\mathscr{B}_{1}$-ring. It then easily follows that the class of $\mathscr{B}_{1}$-rings (which we shall denote by $\left.\mathscr{B}_{1}\right)$ is a radical class.

LEMma 3.2. A nonzero $\mathscr{B}_{1}$-ring without proper divisors of zero is a field of prime characteristic which is algebraic over its prime subfield.

Proof. Let $R$ be a nonzero $\mathscr{B}_{1}$-ring without proper divisors of 
zero. If $x$ is a nonzero element of $R$ there are integers $a_{2}, \cdots, a_{k}$ such that $x=\sum_{i=2}^{k} a_{i} x^{i}$, hence $e_{x}=\sum_{i=2}^{k} \alpha_{i} x^{i-1}$ is an identity for [x]. Since $x$ is not a zero divisor $e_{x}$ is an identity for $R$. If $w \in R, w \neq 0$, $\boldsymbol{e}_{w} \in[w]=[w]^{2}$ so $e_{w} \in[w] \cdot w \subseteq R w$ thus $R=R w$. Since $R$ is nonzero, $R$ is a division ring.

Let $e$ be the identity of $R$. Then $[2 e]=[2 e]^{2}=[4 e]$ so $N e=0$ for some positive integer $N$. Consequently the characteristic of $R$ is a prime and since $e=e_{w} \in[w]$ for all nonzero $w \in R, R$ is algebraic over its prime subfield. Therefore, by Theorem 2, page 183 of Jacobson [7] $R$ is a field.

CoROllary 3.3. If $R$ is a $\mathscr{B}_{1}$-ring then $R$ is isomorphic to a subdirect sum of algebraic fields of prime characteristic. So, in particular, $R$ is commutative.

Proof. If $x \in R, x^{N}=0$ and $R \in \mathscr{B}_{1}$, then $[x]=[x]^{2}=\cdots=[x]^{N}=$ (0) so $x=0$. Hence $\mathscr{B}_{1}$-rings do not have nilpotent elements so the corollary follows from 2.1 and 3.2 .

THEOREM 3.4. A ring $R$ is a $\mathscr{B}_{1}$-ring if and only if every finitely generated subring of $R$ is isomorphic to a finite direct sum of finite fields.

Proof. Let $R \in \mathscr{B}_{1}$ and $R^{\prime}$ be a finitely generated subring of $R$. Then $R^{\prime} \in \mathscr{B}_{1}$ and hence is commutative, so by the Hilbert Basis Theorem $R^{\prime}$ has maximum condition on ideals. If $P^{\prime} \neq R^{\prime}$ and $P^{\prime}$ is a prime ideal of $R^{\prime}$ then $P^{\prime}$ is a maximal ideal of $R^{\prime}$ since by $3.2 R^{\prime} / P^{\prime}$ is a field. Since $R^{\prime}$ is finitely generated, commutative, and $[g]$ has an identity for each generator $g$ of $R^{\prime}, R^{\prime}$ has an identity. Then by Theorem 2, page 203 of [11] $R^{\prime}$ has minimum condition on ideals. But then $R^{\prime}$ is a commutative Wedderburn ring so $R^{\prime}$ is isomorphic to a finite direct sum of fields each of which must be finite since they are finitely generated, algebraic and of prime characteristic.

The converse is obvious; in fact, if $x \in R^{\prime}$ and $R^{\prime}$ is isomorphic to a finite direct sum of finite fields then there is an integer $n(x) \geqq 2$ such that $x^{n(x)}=x$. Thus we have:

CoRollaRY 3.5. $R$ is a $\mathscr{B}_{1}$-ring if and only if for each $x \in R$ there exists an integer $n(x) \geqq 2$ such that $x^{n(x)}=x$.

A class of rings $\mathscr{C}$ is said to be hereditary if $I \triangleleft R \in \mathscr{C}$ implies that $I \in \mathscr{C}$. Analogously we say:

DeFinition 3.6. A class of rings $\mathscr{C}$ is strongly hereditary . $\equiv$ if $S$ is a subring of $R \in \mathscr{C}$ then $S \in \mathscr{C}$. 
Proposition 3.7. If $\mathscr{F}$ is a strongly hereditary finite set of finite fields then a ring $R$ is isomorphic to a subdirect sum of fields in $\mathscr{F}$ if and only if every finitely generated subring of $R$ is isomorphic to a finite direct sum of fields in $\mathscr{F}$.

Proof. Since $\mathscr{F}$ is a finite set of finite fields there exists an integer $N \geqq 2$ such that $x^{N}=x$ for all $x \in F \in \mathscr{F}$.

Let $R$ have ideals $I_{\alpha}: \alpha \in A$ such that $R / I_{\alpha} \cong F_{\alpha} \in \mathscr{F}$ and $\cap\left\{I_{\alpha}: \alpha \in A\right\}=(0)$. Let $R^{\prime}$ be a finitely generated subring of $R$. Then $R^{\prime} \in \mathscr{B}_{1}$ since $x^{N}=x$ for all $x \in R \supseteqq R^{\prime}$, so by $3.4 R^{\prime} \cong A_{1} \oplus \cdots \oplus A_{k}$ and the $A_{i}$ are finite fields. Choose $a_{i} \in R^{\prime}$ such that $\left[a_{i}\right] \cong A_{i}$. Then $a_{i} \neq 0$ so $a_{i} \notin I_{\beta_{i}}$ for some $\beta_{i} \in A$ but $I_{\beta_{i}} \cap\left[a_{i}\right] \triangleleft\left[a_{i}\right]$ so $I_{\beta_{i}} \cap\left[a_{i}\right]=(0)$. Therefore $A_{i} \cong\left[a_{i}\right] \cong\left[a_{i}\right]+I_{\beta_{i}} / I_{\beta_{i}}$ is isomorphic to a subring of $F_{\beta_{i}}$. Since $\mathscr{F}$ is strongly hereditary $R^{\prime}$ is isomorphic to a finite direct sum of fields in $\mathscr{F}$.

Conversely, if every finitely generated subring of $R$ is isomorphic to a finite direct sum of fields in $\mathscr{F}, R$ must be a $\mathscr{B}_{1}$-ring since again $x^{N}=x$ for all $x \in R$. Thus by 3.3 there are ideals $I_{\alpha}: \alpha \in A$ of $R$ such that $\cap\left\{I_{\alpha}: \alpha \in A\right\}=(0)$ and $R / I_{\alpha}$ is a field of prime characteristic; moreover, $R / I_{\alpha}$ must be a finite field since $x^{N}-x=0 \in I_{\alpha}$ for all $x \in R$. Therefore, for each $\alpha \in A$, there exists $x_{\alpha} \in R$ such that $\left[x_{\alpha}\right]+I_{\alpha} / I_{\alpha}=$ $R / I_{\alpha}$. But then $R / I_{\alpha}$ is a homomorphic image of $\left[x_{\alpha}\right]$ so $R / I_{\alpha}$ is isomorphic to a field in $\mathscr{F}$.

\section{Semi-simple radical classes.}

LEMMA 4.1. If $\mathscr{G}$ is a class of rings such that subdirect sums of rings in $\mathscr{C}$ are in $\mathscr{C}$ and $\mathscr{C}$ satisfies $(A)$ then $\mathscr{C}$ is strongly hereditary.

Proof. Let $R \in \mathscr{C}$ and $S$ be a subring of $R$.

Set $R_{i}=R$ for all $i \in Z^{+}=$the set of positive integers. Now the (discrete) direct sum $\sum\left\{R_{i}: i \in Z^{+}\right\}$is an ideal of the direct product (complete direct sum) $\Pi\left\{R_{i}: i \in Z^{+}\right\}$. If $s \in S$ let $\hat{s}(i)=s$ for all $i \in Z^{+}$. Then $S \rightarrow \Delta(S)=\{\hat{s}: s \in S\}$ is an embedding of $S$ into $\Pi\left\{R_{i}: i \in Z^{+}\right\}$. $\Delta(S)+\sum\left\{R_{i}: i \in Z^{+}\right\}$is a subdirect sum of copies of $R$ and hence is in $\mathscr{C}$, so

$$
S \cong \Delta(S) \cong \frac{\Delta(S)+\sum\left\{R_{i}: i \in Z^{+}\right\}}{\sum\left\{R_{i}: i \in Z^{+}\right\}} \in \mathscr{C}
$$

Using a theorem of Amitsur [1] which states that every ring is a homomorphic image of a subdirect sum of total matrix rings of finite order over the ring of all integers, Armendariz in [5] proves 
that if a hypernilpotent radical class $\mathscr{C}$ is a semi-simple class, then $\mathscr{C}$ contains all rings. A hypernilpotent radical class is a hereditary radical class which contains all nilpotent rings.

THEOREM 4.2. If $\&$ is a semi-simple radical class and $\mathscr{E} \nsubseteq \mathscr{P}_{1}$ then $\mathscr{C}$ consists of all rings.

Proof. Let $\mathscr{C}$ be a semi-simple radical class. If $\mathscr{C} \nsubseteq \mathscr{S}_{1}$ then there is a $R \in \mathscr{C}$ and $x \in R$ such that $[x] \neq[x]^{2}$. In [8] Kurosh shows that for any semi-simple class $\mathscr{S}$, subdirect sums of rings in $\mathscr{S}$ are in $\mathscr{S}$. Thus, by $4.1,[x] \in \mathscr{C}$ and since $[x]^{2} \triangleleft[x]$, $[x] /[x]^{2} \in \mathscr{6}$. Now $[x] /[x]^{2}$ is a zero ring on a cyclic group and since $\mathscr{C}$ satisfies $(F), C^{\infty}=$ the zero ring on the infinite cyclic group is in $\mathscr{C}$. This implies (see [3] and [6]) that ' $C$ contains all nilpotent rings. Since $\mathscr{C}$ is a semi-simple class (see [2] and [6]) $\mathscr{C}$ is hereditary, hence $\mathscr{C}$ is hypernilpotent. Therefore, by [5], is the class of all rings.

THEOREM 4.3. If ' $\mathcal{C}$ ' is not the class of all rings then the following are equivalent:

(1) $\mathscr{C}$ is a semi-simple radical class,

(2) there is a strongly hereditary finite set $6(F)$ of finite fields such that: $R \in \mathscr{C}$ if and only if $R$ is isomorphic to a subdirect sum of fields in $6(F)$,

(3) there is a strongly hereditary finite set $G(F)$ of finite fields such that: $R \in \mathscr{C}$ if and only if every finitely generated subring of $R$ is isomorphic to a finite direct sum of fields in $\mathscr{C}(F)$.

Proof. By 3.7 we have that (2) and (3) are equivalent.

Assume that $\mathscr{C}$ satisfies condition (3). Clearly $\mathscr{C}$ satisfies (A) and $(\mathrm{E})$.

If $B \triangleleft A$ and both $A / B$ and $B$ are in $C$ and $A^{\prime}$ is a finitely generated subring of $A$ then $A^{\prime}+B / B \cong A^{\prime} / A^{\prime} \cap B$ is isomorphic to a finite direct sum of fields in $\mathscr{C}(F)$. A slight modification of the proof given for Proposition 1 on page 241 of Jacobson [7] shows that $A^{\prime} \cap B$ is finitely generated as a ring. Thus $A^{\prime} \cap B$ is also isomorphic to a finite direct sum of fields in $\mathscr{C}^{\prime}(F)$ and so $A^{\prime} \cong A^{\prime} / A^{\prime} \cap B \oplus A^{\prime} \cap B$. Therefore $A \in \mathscr{C}$. From this it is easy to show that if $\mathscr{C}^{\prime}(R)=$ the sum of all ideals of $R$ which are in $\mathscr{C}$ then $\mathscr{C}(R) \in \mathcal{C}$ and $\mathscr{C}(R / \mathscr{C}(R))=(0)$. Thus, $\mathscr{C}$ satisfies $(\mathrm{B})$ and $(\mathrm{C})$.

If every nonzero ideal of a ring $R$ can be homomorphically mapped onto a nonzero ring in $\mathscr{C}$ then by 3.7, every nonzero ideal of $R$ can be homomorphically mapped onto a ring in $\mathscr{C}(F)$. Sulinski [9] (see also [6], Theorem 46) shows that this implies that $R$ is isomorphic to a subdirect sum of rings in $\mathscr{C}(F)$ and hence by 3.7 again, $R \in \mathscr{C}$. So 
$\mathscr{C}$ satisfies $(\mathrm{F})$ and hence $\mathscr{C}$ is a semi-simple radical class.

Conversely, suppose $\mathscr{C}$ satisfies condition (1). Let $\mathscr{C}(F)=$ the class of all fields which are in $\mathscr{C}$ and define $A=\Pi\{R: R \in \mathscr{C}(F)\}$. Since $\mathscr{C}$ is a semi-simple class subdirect sums of rings in $\mathscr{C}$ are in $\mathscr{C}$; thus $A \in \mathscr{C}$. By hypothesis, $\mathscr{C} \subseteq \mathscr{Q}_{1}$ so by 3.4 all elements of $A$ must be torsion. From this it follows that there is a finite number of primes $p_{1}, \cdots, p_{N}$ such that every field in $\mathscr{C}(F)$ is of characteristic $p_{i}$ for some $1 \leqq i \leqq N$. For each finite field $R \in \mathscr{C}(F)$ choose $a(R)$ such that $[a(R)]=R$ and for each infinite field $R \in \mathscr{C}(F)$ set $a(R)=0$. Then $a=\{a(R)\}_{R \in \dot{c}(R)}$ is in $A$ and by $3.5 a^{K}=a$ for some integer $K \geqq 2$. Thus, for all finite fields $R$ in $\mathscr{C}(F)$, the dimension of $R$ over its prime subfield is $\leqq K-1$. Hence there is only a finite number of finite fields in $\mathscr{C}(F)$. Suppose there is an infinite field $R \in \mathscr{C}(F)$. By $3.2 R$ is of prime characteristic and is algebraic over its prime subfield so $R$ has an infinite number of non-isomorphic finite subfields. All these subfields are in $\mathscr{C}(F)$ since $\mathscr{C}$ is strongly hereditary by 4.1 . This is impossible since there is only a finite number of finite fields in $\mathscr{C}(F)$. Therefore $\mathscr{C}(F)$ is a strongly hereditary finite set of finite fields. If $R \in \mathscr{C}$ then $R \in \mathscr{B}_{1}$ so by $3.3 R$ is isomorphic to a subdirect sum of fields all of which are in $\mathscr{C}(F)$ since $\mathscr{C}$ satisfies (A). Conversely, any ring isomorphic to a subdirect sum of rings in $\mathscr{C}(F)$ is in $\mathscr{C}$ since $\mathscr{G}$ is semi-simple class. Thus $\mathscr{C}$ satisfies (2).

\section{REFERENCES}

1. S. A. Amitsur, The identities of P. I.-rings, Proc. Amer. Math. Soc. 4 (1953), 27-34.

2. T. A. Anderson, N. Divinsky, and A. Sulinski, Hereditary radicals in associative and alternative rings, Canad. J. Math. 17 (1965), 594-603.

3. - Lower radical properties for associative and alternative rings, J. London Math. Soc. 41 (1966), 417-24.

4. V. Andrunakievic, Radicals in associative rings II, Mat. Sb. 55 (1961), 329-46.

5. E. P. Armendariz, Closure properties in radical theory, Pacific J. Math. 26 (1968), 1-8.

6. N. J. Divinsky, Rings and radicals, Univ. of Toronto Press, Toronto, 1965.

7. N. Jacobson, Structure of rings, Amer. Math. Soc. Coll. Publ. 37 (1964).

8. A. G. Kurosh, Radicals of rings and algebras, Mat. Sb. 33 (1953), 13-26.

9. A. Sulinski, Certain questions in the general theory of radicals, Mat. Sb. 44 (1958), 273-86.

10. G. Thierrin, Sur les ideaux complement premiers d'un annaux quelconque, Bull. Acad. Roy. Belg. 43 (1957), 124-32.

11. O. Zariski and P. Samuel, Commutative algebra, Vol. I, Van Nostrand ,Princeton N. J., 1958.

Received November 6, 1968. The author holds a National Research Council of Canada Postgraduate Scholarship.

UNIVERSity of BRITISH COLUMBIA 


\section{PACIFIC JOURNAL OF MATHEMATICS}

\section{EDITORS}

\author{
H. SAMELSON \\ Stanford University \\ Stanford, California 94305 \\ Richard Pierce \\ University of Washington \\ Seattle, Washington 98105
}

J. DUGUNDJI

Department of Mathematics

University of Southern California

Los Angeles, California 90007

BASIL GORDON*

University of California

Los Angeles, California 90024

\section{ASSOCIATE EDITORS}
E. F. BECKENBACH
B. H. NeUmanN
F. WOLF
K. YoSHIDA

\section{SUPPORTING INSTITUTIONS}

UNIVERSITY OF BRITISH COLUMBIA CALIFORNIA INSTITUTE OF TECHNOLOGY

UNIVERSITY OF CALIFORNIA MONTANA STATE UNIVERSITY

UNIVERSITY OF NEVADA

NEW MEXICO STATE UNIVERSITY

OREGON STATE UNIVERSITY

UNIVERSITY OF OREGON

OSAKA UNIVERSITY

UNIVERSITY OF SOUTHERN CALIFORNIA
STANFORD UNIVERSITY

UNIVERSITY OF TOKYO

UNIVERSITY OF UTAH

WASHINGTON STATE UNIVERSITY

UNIVERSITY OF WASHINGTON

AMERICAN MATHEMATICAL SOCIETY CHEVRON RESEARCH CORPORATION TRW SYSTEMS

NAVAL WEAPONS CENTER 


\section{Pacific Journal of Mathematics}

\section{Vol. 32, No. $1 \quad$ January, 1970}

Robert Alexander Adams, Compact Sobolev imbeddings for unbounded domains ........................................ 1

Bernhard Amberg, Groups with maximum conditions .................. 9

Tom M. (Mike) Apostol, Möbius functions of order k............... 21

Stefan Bergman, On an initial value problem in the theory of two-dimensional transonic flow patterns ................... 29

Geoffrey David Downs Creede, Concerning semi-stratifiable spaces ...... 47

Edmond Dale Dixon, Matric polynomials which are higher

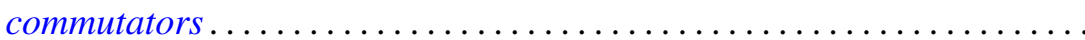

R. L. Duncan, Some continuity properties of the Schnirelmann density.

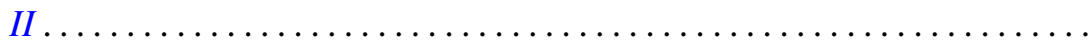

Peter Larkin Duren and Allen Lowell Shields, Coefficient multipliers of $H^{p}$

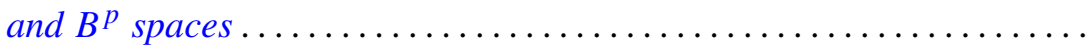

Hector O. Fattorini, On a class of differential equations for vector-valued

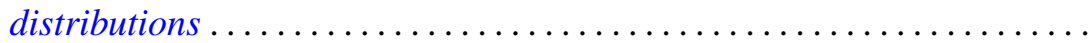

Charles Hallahan, Stability theorems for Lie algebras of derivations. . . . . . 105

Heinz Helfenstein, Local isometries of flat tori ................ 113

Gerald J. Janusz, Some remarks on Clifford's theorem and the Schur

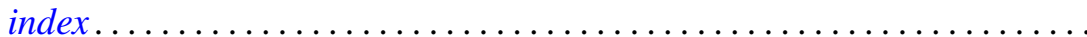

Joe W. Jenkins, Symmetry and nonsymmetry in the group algebras of discrete groups. ...

Herbert Frederick Kreimer, Jr., Outer Galois theory for separable

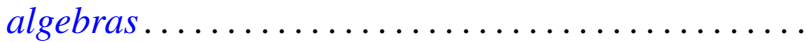

D. G. Larman and P. Mani, On visual hulls

R. Robert Laxton, On groups of linear recurrences. II. Elements of finite order.

Dong Hoon Lee, The adjoint group of Lie groups ...

James B. Lucke, Commutativity in locally compact rings

Charles Harris Scanlon, Rings of functions with certain Lipschitz

$$
\text { properties ............................... }
$$

Binyamin Schwarz, Totally positive differential systems .

James McLean Sloss, The bending of space curves into piecewise helical curves.

James D. Stafney, Analytic interpolation of certain multiplier spaces ...

Patrick Noble Stewart, Semi-simple radical classes.......

Hiroyuki Tachikawa, On left $\mathrm{QF}-3$ rings ...................... 255

Glenn Francis Webb, Product integral representation of time dependent nonlinear evolution equations in Banach spaces.... . . . 